\title{
Efficiency of a Solar Hydronic Space Heating System under the Algerian Climate
}

\author{
Ilhem Zeghib \\ Energy Physics Laboratory \\ Department of Physics, Brothers Mentouri University \\ Constantine, Algeria \\ Imita75@yahoo.fr
}

\author{
Abla Chaker \\ Energy Physics Laboratory \\ Department of Physics, Brothers Mentouri University \\ Constantine, Algeria \\ Chakamine@yahoo.fr
}

\begin{abstract}
Hydronic heating systems supplied by renewable energy sources are one of the main solutions for substituting fossil fuel and natural gas consumption. This paper presents the development of modeling and analysis of a solar hydronic heating system in an existing single-family house built in 1990's heated by low-temperature radiators. The simulation has been used to study the potential of using this system under climatic conditions in Algeria. And for this purpose, a component based on the simulation model for the thermal behavior of each component of the system are carried out in order to evaluate the economic performance for this system. The system is compared, with a conventional high-temperature boiler system. The results indicated that single-family houses could be heated with solar hydronic heating and provided an acceptable level of thermal comfort in the room with $22^{\circ} \mathrm{C}$, according to the results of the analysis, the solar energy covers only $20.8 \%$ of the total energy consumption in a single-family house. Furthermore, the thermal performance of the heating conventional system can be largely improved up to $15 \%$.
\end{abstract}

Keywords-solar collector; low temperature heating; solar heating; indoor temperature; efficiency

\section{INTRODUCTION}

In Algeria, energy consumption in the building sector accounts for almost $40 \%$ of the total final energy use [1] with heating and hot water being responsible for almost $60 \%$ of that [1]. This high consumption has led to opt for new low-energy buildings and to retrofit old ones, and take measures such as using additional insulation, tight building envelopes, or energysaving equipments. All these measures help reduce the season and space heating load and provide an opportunity to use lowtemperature heating systems. These systems usually work with a maximum supply water temperature of $45^{\circ} \mathrm{C}$. So a new generation of hydronic heating systems operating at low temperatures and employing renewable energy sources such as geothermal sources and solar energy has emerged.

In [2], authors show that renewable sources of heat can be integrated into the district heating system without problems and contribute to the fossil-free heating sector. In [3], the author studied the technical and economical potential to use solar energy in the Finnish district heating system. The function of a district heating system connected to the solar collector field was simulated with the TRNSYS (Transient System Simulation Tool) software. The results show that solar collectors could provide the $10 \%$ of the yearly heat production. In the existing single-family house, we can make very significant savings by replacing or supplementing an old system with a solar heating system. The objective is to meet the heating needs using the least possible energy auxiliary, valorize a performance enhancement of solar energy. For this purpose, integration of the solar thermal system should be relatively simple: all that will need to be done is to replace the boiler of a traditional heating fluid with a storage tank heated with a set of solar panels and existing conventional radiators with oversized radiators (low temperature). In this paper, we are studying the dynamic behavior of the solar hydronic space system is represented by a mathematical model corresponding to an energy balance of each element of the solar system, collector, tank and the radiators.

\section{SYSTEM DESCRIPTION}

\section{A. Heating system}

A schematic diagram of the solar hydronic space heating system used in the present study is shown in Figure 1. The solar space heating system consists of the flat plate collectors, a hot water storage tank, the piping, the controllers, and the auxiliary heating system [4]. The load distribution system consists of low temperature radiators and two pumps to transfer the energy to the storage and to the load. The circulating water from the collector transmits its heat to the storage tank water where it is stored in the form of a sensitive heat until it can be used. An auxiliary heater (gas boiler) is connected to the storage tank to supplement solar heating, when needed to meet temperature requirement of the load. In our study, the heat production system is the combination of solar collectors and a gas boiler. When water temperature in the storage tank is high enough for space heating, solar collectors are used as the only heat source can deliver hot water directly to the low temperature radiators. In this respect, auxiliary heater operates only when the temperature of the water in the tank is lower than $45^{\circ} \mathrm{C}$ which will reduce the boiler energy. 


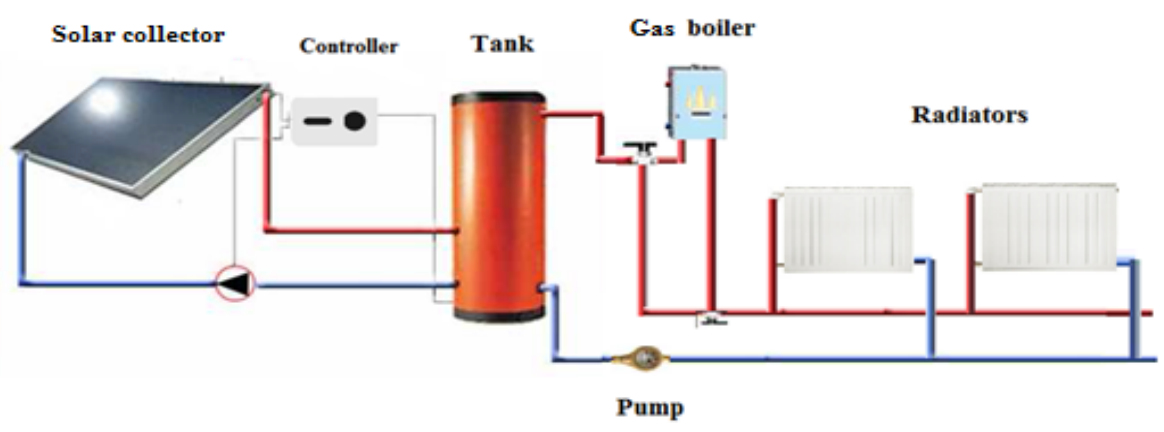

Fig. 1. Schematic diagram of solar low heating system

\section{B. Hydronic distribution system}

The component parts of a solar-water distribution system are similar to that of a conventional heating system. The hydronic systems use pipes and circulation pumps to distribute heated water throughout the house. A heat distribution grid can transfer heat from the tank to the low-temperature radiator. The low-temperature hydronic heating concept can be extended to existing houses by replacing existing radiators with low temperature radiators and changing the design operating conditions from $70 / 60 / 20^{\circ} \mathrm{C}$ to $50 / 40 / 20^{\circ} \mathrm{C}$ [3]. In our study, the length and height of the radiators are kept the same but their depth is changed. This meant that while all the original radiators were type 21, the low-temperature radiators were type 33 [5]. As such, replacing the radiators is very easy because the radiators can be connected to the existing piping system without any changes.

\section{Modeling System COMPONENTS}

The simulation procedure involves casting mathematical models for each system component and then combining these models consecutively to accomplish the complete simulation. Thermal models compute each component exit temperature (collector, storage tank, low temperature radiator) and system performance, were estimated every minute.

\section{A. Thermal analysis}

\section{1) Solar Collector}

Two flat-plate collectors, a $1.5 \mathrm{~m}^{2}$ gross surface area each, were used for the collection of solar energy. The collectors have been installed facing south and inclined at an angle of $45^{\circ} \mathrm{C}$ from the horizon for maximum gain during winter collection. The Mathematical Model describes the flat-plate solar collector system considering the transient properties of its different zones. In the proposed model, the analyzed control volume of the flat-plate solar collector contains one tube that is divided into five nodes (glass cover, air gap, absorber, fluid and insulation) perpendicular to the liquid flow direction.

The collector efficiency is defined as the ratio of usable heat energy extracted from a collector by the heat transfer fluid during any time period to the solar energy striking the cover during that same time period [6]:

$$
\eta_{c}=\frac{\int_{t_{1}}^{t_{2}} Q_{u} d t}{A_{c} \int_{t_{1}}^{t_{2}} I_{g} d t}
$$

\section{2) Water storage tank}

A stratified storage tank was used in this simulation, a mathematical model for heat transfer in the storage tank based on the one-dimensional transient heat transport equation by convection and conduction along the prevailing flow direction of the storage tank. In multi-node modeling, the tank is divided into $\mathrm{N}$ nodes or sections, with energy balances written for each node. The energy equation takes into account the energy gain from the collector, the energy loss in the surrounding, and utilized by the load. This results in $\mathrm{N}$ differential equations to be solved simultaneously to obtain the temperature of each node [7]. In our study the tank is divided into 150 nodes.

Thermal efficiency at tank [9]:

$$
\eta_{S}=\frac{Q_{d i s}}{Q_{S}}
$$

Where $Q_{\text {dis }}$ is the heat transferred to the distribution system is defined by:

$$
Q_{\text {dis }}=Q_{s}-Q_{\text {loss }, s}
$$

The energy storage in the tank is related to the mass and the difference between the initial and final temperature of water in the storage tank can be expressed as [10]:

$$
Q_{s}=\frac{1}{n} \sum_{i=1}^{n} m_{s, i} c p_{s, i}\left(T_{s, i+1}-T_{s, i}\right)
$$

The heat losses from the tank is given by [11]

$$
Q_{\text {loss }, s}=\frac{1}{n} \sum_{i=1}^{n} U_{s, i} A_{s, i}\left(T_{s, i}-T_{a}\right)
$$

3) Auxiliary heating system

The auxiliary heating system used in conjunction with a solar heating system is of the plain/traditional type. Although 
the auxiliary boiler can be controlled in different ways, normally the auxiliary heater in series would be used to the temperature of the water from storage only when the water temperature in the storage tank is too low to meet the heating requirement in the house, and has a desired temperature of $45^{\circ} \mathrm{C}$.

The auxiliary efficiency was calculated as :

$$
\eta_{a u}=\frac{Q_{a u}}{Q_{b u r}}=\frac{\dot{m} C p\left(T_{s o}-T_{s, 1}\right)}{m_{C a z} \cdot P C I_{G a z}}
$$

$Q_{a u}$ The useful energy transmitted by the auxiliary to the hot water, $Q_{b r u}$ the energy used by the burner.

\section{4) Pipe heating}

The task of the distribution system is to connect all various components of the heating System. The pipes distribute a heating medium lose some it to the surroundings. This heat loss causes undesired cooling of the medium in the pipes; how they can be acceptable if the pipes are placed within a heated space. However, this form of heat transfer is undesirable, and it cannot be regulated and may not be required for most of the time.

The efficiency of the pipes heating:

$\eta_{P I}=\left\{\begin{array}{lll}\frac{Q_{p}}{Q_{d i s}}=1-\frac{Q_{\text {loss }, p}}{Q_{d i s}} & \text { if } & T_{s, 1}>45^{\circ} \mathrm{C} \\ \frac{Q_{p}}{Q_{a u}}=1-\frac{Q_{\text {loss }, p}}{Q_{a u}} & \text { if } & T_{s, 1}<45^{\circ} \mathrm{C}\end{array}\right.$ [12]:

The heat emissions of pipes are calculated is following

$$
Q_{\text {loss }, p}=L_{C} \cdot Y_{C} \cdot\left(T_{m f}-T_{n}\right)
$$

Where $Y_{c}$ is the linear thermal transmittance, $L_{c}$ is the total length of pipes. $T_{m f}$ is the pipes inner temperature and $T_{n}$ is the interior temperature.

\section{5) Low-temperature radiators}

The level of water temperature supplied to the heat emitter in buildings plays a major role in primary energy consumption. The main principle of a low-temperature heating system is to provide the same thermal comfort as a medium-temperature heating system, while using a lower supply temperature. The radiators for low temperature systems are physically and technically the same as traditional panel radiators. The only key factor which changes is sizing. Normally manufacturers' data sheets will quote radiator output when there is a temperature difference (water to air). If a radiator is required to run at a lower temperature than normal, its size must be increased to compensate for this temperature difference.

The water return temperature through radiators based on actual heat emission and inlet water temperatures is calculated by [13]:

$$
C_{r} \frac{d T_{r s}}{d t}=\dot{m}_{r} C_{p}\left(T_{r e}-T_{r s}\right)+(U A)_{r}\left(T_{b}-T_{r s}\right)
$$

The emission efficiency is giving by :

$$
\eta_{R E}=\frac{Q_{R E}}{Q_{p}}
$$

\section{6) Eenergy demand in the house}

The energy balance of a house is characterized by energy losses and gains, energy losses are transmission and ventilation and these losses can be fully or partly compensated by energy gains. Different sources of energy gains can be utilized, such as internal energy gains caused by appliances and users as well as solar gains through openings. In this study, the energy need of the house during the heating is estimated using the equations in thermal regulation for Algerian buildings. The need of useful heat depends on thermal qualities of its envelope (thermal resistances), and its losses by ventilation.

The energy need for heating is giving by [14]:

$$
Q_{h}=Q_{l o s}-n \cdot Q_{G}
$$

where $Q_{l o s}$ is the total heat transfer for heating mode, $Q_{G}$ are the total heat gains for the heating, $n$ is the dimensionless gain utilization factor.

The house is modeled as a multi-zone model (each room is modeled as an individual zone). The rooms are heated at a uniform indoor temperature at all times. The room can be modeled as a single heat capacity element. A differential equation is then written relating the heat flow to the room to time derivative of the indoor temperature and the house heat capacity.

The indoor temperature of room is calculated by:

$$
\frac{d T_{b}}{d t}=\frac{1}{C_{b}} Q_{n e t}=\frac{1}{C_{b}}\left(Q_{\text {sup }, b}-Q_{\text {los }, b}\right)
$$

\section{B. Energy performance analysis}

The performance indices of energy evaluated in this study include: energy collected, energy delivered and supply pipe losses, solar fraction, collector efficiency and system efficiency.

\section{1) Solar fraction}

The solar fraction is the amount of energy provided by the solar technology divided by the total energy required. It can be calculated from the equation below [15]:

$$
S F==\frac{\sum Q_{s}}{\sum Q_{s}+\sum Q_{\text {aux }}}
$$

where $Q_{s}$ the solar energy produced, $Q_{a u x}$ auxiliary heating requirement. 


\section{2) System efficiency}

The efficiency of heating systems is increasingly important because of the increasing need to save energy. The major characteristic parameter for estimating the efficiency of the hydronic heating is heat loss factor. The main parts of a heating system, generation, storage, distribution and emission, all have some losses. The distribution losses caused by pipes in unheated area are calculated as non-recoverable losses and losses in heated rooms contribute as recoverable losses. Emission losses consist of heat loss due to non-uniform temperature distribution, heat loss due to heat emitter position and heat loss due to control indoor temperature.

The tolal systems efficiency is calculated dy [16]

$$
\eta_{\text {inst }}=\eta_{S} \cdot \eta_{A U} \cdot \eta_{P I} \cdot \eta_{R E}
$$

\section{RESUltS AND DISCUSSION}

The thermal performance of a solar space heating system is usually estimated by computer simulation, taking into consideration local climatic conditions and energy load. The simulation input parameters include climatic conditions of Adrar $(27.10 \mathrm{~N}, 0.17 \mathrm{E}$, Altitude: $279 \mathrm{~m})$ in Algeria. The weather data are obtained from in-field measurements, using a weather station, the measured radiation and ambient temperature for 1 min intervals over a $96 \mathrm{~h}$ optimization interval are shown in Figure 2. A calculation code is set up and developed in FORTRAN language for the resolution of the thermal balance equations for different component of the solar space heating. The program determine every minute the temperature of the collector, the storage tank, the radiators and energy needed for heating. For the hydronic system, we determine the pipe heat losses, the global efficiency of the solar space heating system. The system was analyzed and optimized over a period of four days from 16th to 19th February.

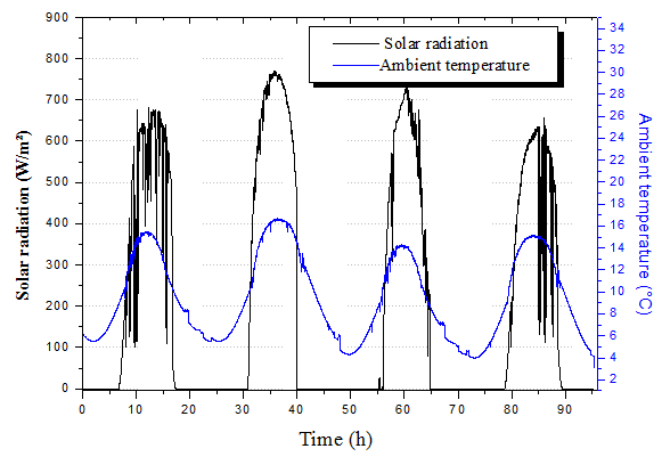

Fig. 2. Variation of solar radiation

In this study, the heated water from solar collectors is stored in a tank for a week without use. The goal is to increase the tank temperature to $55^{\circ} \mathrm{C}$, after we used the water from that tank for heats the space water radiators. Figure 3 shows the variation of the outlet water temperature in the tank and inlet and outlet temperature of the radiators. When water temperatures at the outlet of tank is less than $45^{\circ} \mathrm{C}$, water goes to auxiliary heater to be heated and to be send to the radiators.
This occurs when the solar radiation is not sufficient to heat and if the water storage temperature is over $45^{\circ} \mathrm{C}$ the radiators are heated by hot water from the storage tank.

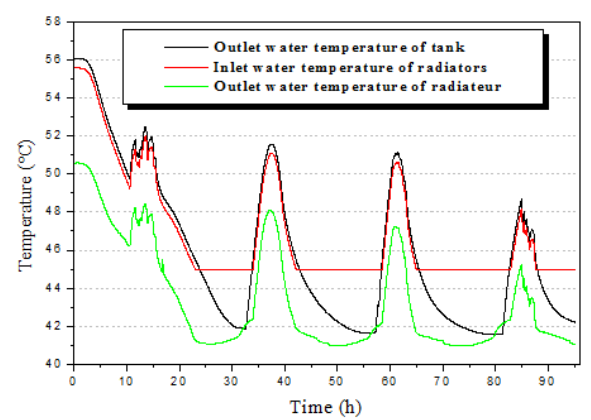

Fig. 3. Water temperature of the radiators and the storage tank

In Figure 4, the energy need for heating of the house is shown for 4 days. It is noticed that heating energy profile is quite similar to ambient temperature profile. In general, heating energy and the ambient temperature are inversely proportional to each other: the higher the heating energy the lower ambient temperature, and vice versa. It may be noted that during the night the heating needs are greater in the living room that two room, its maximum value of $1.2 \mathrm{~kW}$. There is a slight difference of the heating needs in the three rooms between $14.00 \mathrm{~h}$ and $10.00 \mathrm{~h}$ this is obviously caused by effect of the solar gains on the internal conditions, which are different according to orientation of the room.

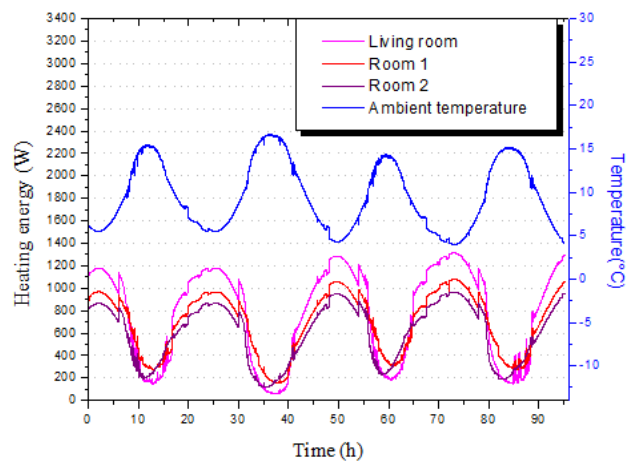

Fig. 4. Variation of energy need for heating during the 4 days

From Figure 5, it has been observed clearly that daily solar fraction is maximal when the storage temperature is higher than $45^{\circ} \mathrm{C}$, the radiators are heated by hot water from the storage tank. When the temperature is below $45^{\circ} \mathrm{C}$, the auxiliary heater is activated, and daily solar fraction starts to decrease with decreasing storage temperature. As it can be observed from Figure 6 the monthly solar fraction decreases from November to January and then increasing until March. During November and March, the solar energy is relatively low but it this coincides with heating requirements which are low. During December and January, the solar fraction decreases and at the same time the contribution of the solar thermal systems to the supply of energy is reduced. Moreover 
it was estimated that the annual solar fraction for this system is about $20.8 \%$.

Figure 7 illustrates the variation of the indoor temperatures in the rooms in the house (living room, room1 and room 2) for four days. It should be noted that the internal temperature profile is quite similar to ambient temperature profile, the temperature is maximum in the middle of the day and minimum for the night, or heating needs are important. They vary between $17^{\circ} \mathrm{C}$ and $23^{\circ} \mathrm{C}$ for three rooms, for a minimum ambient temperature at $7^{\circ} \mathrm{C}$. There is a slight difference temperature of living room and two rooms between $10.00 \mathrm{~h}$ and $14.00 \mathrm{~h}$, this is due to the fact that the living room main facade faces south while the two rooms are oriented to the north.

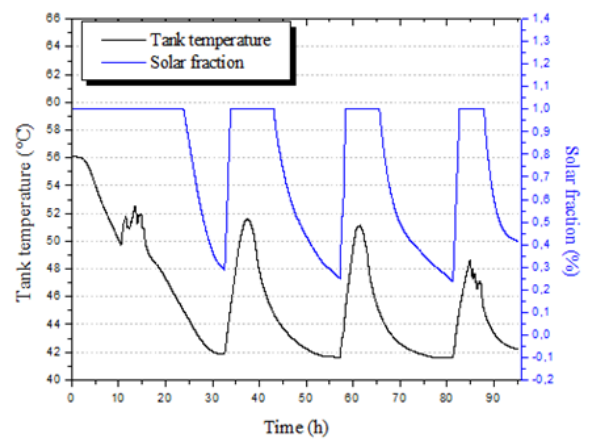

Fig. 5. Variation of daily solar fraction and tank temperature

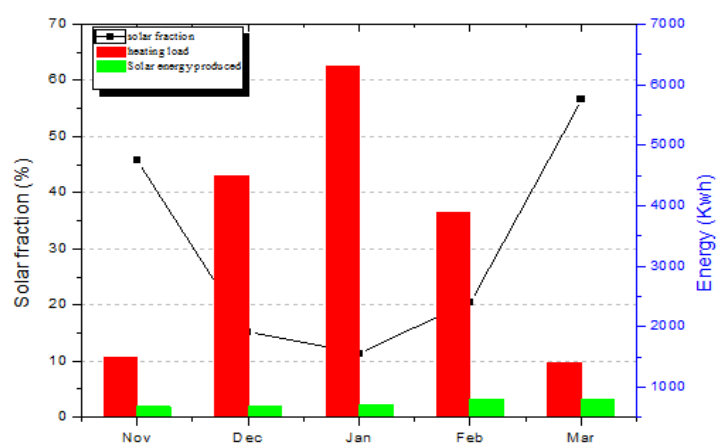

Fig. 6. Variation of monthly solar fraction, annual energy needs and solar energy produced

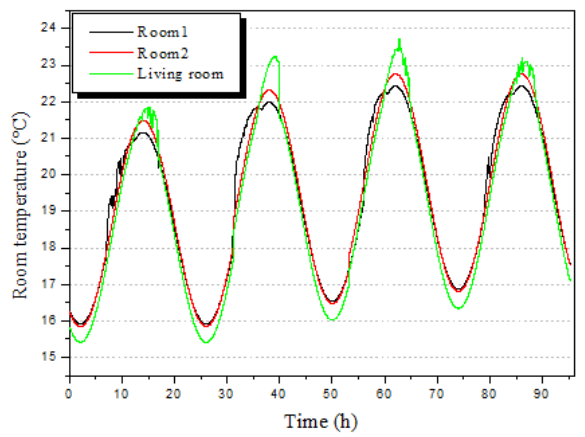

Fig. 7. Room air temperature during 4 days
Figure 8 shows a comparison of indoor room temperature in the room 1 between solar heating and conventional heating. It is observed that temperatures are similar (difference of $0.5^{\circ} \mathrm{C}$ ) and they range between 16 and $23^{\circ} \mathrm{C}$. We can deduce that Solar heating systems can provide a ideal temperature inside the house. Figure 9 shows a comparison of total system efficiency between solar heating and conventional heating. We note that the performance of solar heating systems is higher than conventional heating systems by $15 \%$.This seems logical when the heating system is too hot, the head losses from production units, distribution pipes and radiators are important, therefore, their performance decreases. It should be noted that the system efficiency profile is quite similar to indoor room temperature profile, the system efficiency is maximum in the middle of the day and minimum for the night.

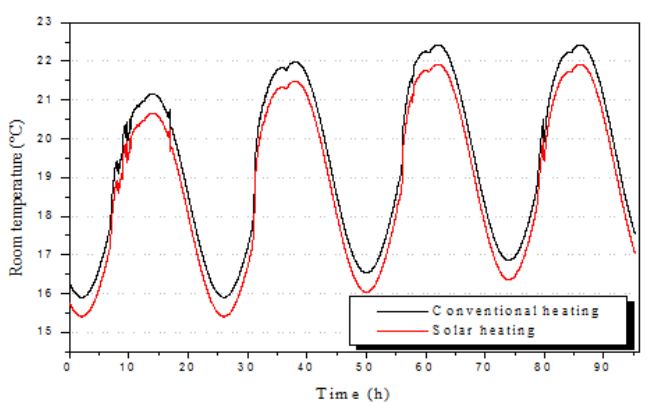

Fig. 8. Comparison of indoor room temperature between conventional and solar heating

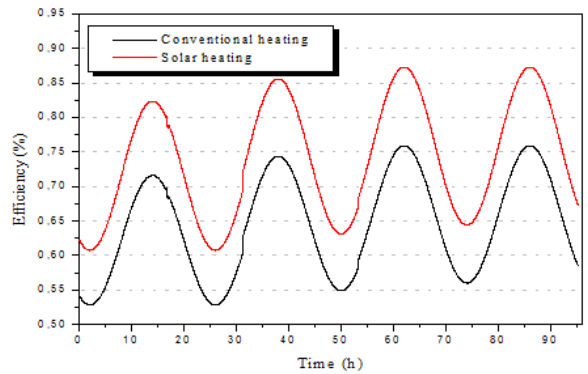

Fig. 9. Comparison of space heating efficiency between conventional and solar heating

The potential savings offered by solar thermal systems are difficult to calculate exactly and depend on a large range of factors. These include: Initial system cost (depending on size, quality of parts and installation), the energy source that you are replacing (coal, gas). Solar heating systems usually cost more to purchase and install than conventional water heating systems. In this study the total cost of the solar heating system was 300000 DA. This figure includes the cost of installation and all parts (solar collectors, controller, pipes, hot water tank). However, regulators and piping distribution system are not included in the calculation of costs as they are part of conventional system. The annual saving offered by this system in the first year of operation is about 3661 kilowatts hours / year, corresponding to $366 \mathrm{~m}^{3}$ of natural gas. We can deduce that the space solar heating in Algeria offer modest savings, because the per unit cost of gas is fairly cheap and the prices 
for natural gas exported by Algeria are 20 times less expensive than the international prices (equal to 4.191DA / KWh (1 Euro $=122.43$ DA )).

\section{CONCLUSION}

System modeling and computer simulations are employed in order to investigate the potential of using a solar hydronic space heating system under the Algerian climate. The developed mathematical analysis is utilized to study the performance of the proposed system. Results showed that this system provide on average, more than $20.8 \%$ of a house's heating energy. Furthermore, the thermal performance of the heating conventional system can be largely improved to $15 \%$. Moreover, the system was capable of sustaining a stable and comfortable indoor temperature in rooms with $22^{\circ} \mathrm{C}$. The results indicate that, considering the cost of solar heating systems and the fuel prices today, a hydronic heating system that integrates solar heating is not yet cost-effective in the Algerian circumstances, especially as the expected pay-back periods tend to be very long. This result proves that this technology is preferred for countries with higher gas price.

\section{REFERENCES}

[1] K. Imessad, R. Kharchi, "Experimental study of a combined solar system for floor heating”, Renewable Energy, Vol. 18, No. 3, pp. 399405, 2015

[2] M. Brand, S. Svendsen, "Renewable-based low-temperature district heating for existing buildings in various stages of refurbishment", Energy , Vol. 62, pp. 311-319, 2013

[3] M. Leskinen, "The use of solar energy in a district heating system in Finland : case study of six district heating plants", New and Renewable Technologies for Sustainable Development, pp. 313-324, 2002

[4] M. Bojic, S. Kalogirou, K. Petronijevic, "Simulation of a solar domestic water heating system using a time marching mode", Renewable Energy, Vol. 24, pp. 441-452, 2002

[5] www. Chappee.com, 2015

[6] Y. Kim, K. Thu, "Thermal analysis and performance optimization of a solar hot water plant with economic evaluation”, Solar Energy, Vol. 86, pp. 1378-1389, 2012

[7] Z. F. Li, K. Sumathy, "Performance study of a partitioned thermally stratified storage tank in a solar powered absorption air conditioning system”, Applied Thermal Engineering, Vol. 22, pp. 1207-1216, 2002

[8] I. Zeghib, A. Chaker, "Modeling and simulation of a solar thermal system for domestic space heating using radiators low temperature", International Journal of Renewable Energy Research, Vol. 1, No. 5, pp. 266-276, 2015

[9] C. J. Porras-Prieto, F. R. Mazarron, "Influence of required tank water temperature on the energy performance and water withdrawal potential of a solar water heating system equipped with a heat pipe evacuated tube collector”, Solar Energy, Vol. 110, pp. 365-377, 2014

[10] M. Arslan, "Thermal performance of a vertical solar hot water storage tank with a mantle heat exchanger depending on the discharging operation parameters”, Solar Energy, Vol. 116, pp. 184-204, 2015

[11] H. Wang, C. Qi, "Performance study of underground thermal storage in a solar-ground coupled heat pump system for residential buildings", Energy and Buildings, Vol. 40, pp. 1278-1286, 2008

[12] S. Ntsaluba, B. Zhu , "Optimal flow control of a forced_circulation solar water heating system with energy storage units and connecting pipes", Renewable Energy, Vol. 89, pp. 108-124, 2016

[13] L. Haiyan, P. Valdimarsson Lamberto, "District heating modelling and simulation", 34th Workshop on Geothermal Reservoir Engineering, 2009
[14] L. Tronchin, K. Fabbri, "Energy performance building evaluation in Mediterranean countries: Comparison between software simulations and operating rating simulation”, Energy and Buildings., Vol. 42, pp. 18621877, 2010

[15] E. Kazanavicius, A. Mikuckas, "The heat balance model of residential house”, Information Technology and Control , Vol. 35, pp. 391-396, 2006

[16] M. Maivel, J. Kurnitski, "Low temperature radiator heating distribution and emission efficiency in residential buildings", Energy and Buildings, Vol. 69, pp. 224-236, 2014 